Kragujevac Journal of Mathematics

Volume 45(6) (2021), Pages 969-975.

\title{
THE MINIMUM EDGE COVERING ENERGY OF A GRAPH
}

\author{
SAMIRA SABETI ${ }^{1}$, AKRAM BANIHASHEMI DEHKORDI ${ }^{1}$, \\ AND SAEED MOHAMMADIAN SEMNANI ${ }^{1}$
}

\begin{abstract}
In this paper, we introduce a new kind of graph energy, the minimum edge covering energy, $E_{C_{E}}(G)$. It depends both on the underlying graph $G$, and on its particular minimum edge covering $C_{E}$. Upper and lower bounds for $E_{C_{E}}(G)$ are established. The minimum edge covering energy and some of the coefficients of the polynomial of well-known families of graphs like Star, Path and Cycle Graphs are computed.
\end{abstract}

\section{INTRODUCTION}

In the study of spectral graph theory, we use the spectra of the certain matrix associated with the graph, such as the adjacency matrix, the Laplacian matrix, and other related matrices. Some useful information about the graph can be obtained from the spectra of these various matrices. Let $G$ be a simple graph and let its vertex set be $V(G)=\left\{v_{1}, v_{2}, \ldots, v_{n}\right\}$. The adjacency matrix $A(G)$ of the graph $G$ is a square matrix of order $n$ whose $(i, j)$-entry is equal to unity if the vertices $v_{i}$ and $v_{j}$ are adjacent and it is equal to zero otherwise. The eigenvalues $\lambda_{1}, \lambda_{2}, \ldots, \lambda_{n}$ of $A(G)$, assumed in nonincreasing order, are the eigenvalues of the graph $G$. The concept of energy of a graph was introduced by I. Gutman [1] in the year 1978 as the sum of the absolute values of its eigenvalues:

$$
E(G)=\sum_{i=1}^{n}\left|\lambda_{i}\right|
$$

One of the remarkable chemical applications of spectral graph theory is based on the close correspondence between the graph eigenvalues and the molecular orbital energy

Key words and phrases. Minimum edge covering set, minimum edge covering matrix, graph energy, minimum edge covering eigenvalues.

2018 Mathematics Subject Classification. Primary: 05C50.

DOI 10.46793/KgJMat2106.969S

Received: November 19, 2018.

Accepted: July 15, 2019. 
levels of $\pi$-electron in conjugated hydrocarbons. An interesting quantity in Huckel theory is the sum of the energies of all the electrons in a molecule, so-called total $\pi$-electron energy $E$. For more details on the mathematical aspects of the theory of graph energy see [2-5]. The basic properties including various upper and lower bounds for energy of a graph have been established [6,7], and it has found remarkable chemical applications in the molecular orbital theory of conjugated molecules [8]. Recently C. Adiga et al. [8] defined the minimum covering energy, $E_{C}(G)$ of a graph which depends on its particular minimum cover $C$. Further, incidence energy, matching energy, minimum dominating energy, Laplacian minimum dominating energy and minimum dominating distance energy of a graph $G$ can be found in [9-16]. Motivated by these papers, we study the minimum edge covering $E_{C_{E}}$ of a graph $G$. We compute some properties of the characteristic polynomial of a minimum edge covering matrix of a graph $G$. Upper and lower bounds for $E_{C_{E}}(G)$ are established. It is possible that the minimum edge covering energy that we are considering in this paper may have some applications in chemistry as well as in other areas. Let $G=(V, E)$ be a simple finite graph that is has no loops no multiple and directed edges. Graph $G$ has $n$ vertices, $m$ edges with vertex set $V(G)=\left\{v_{1}, v_{2}, \ldots, v_{n}\right\}$ and edge set $E(G)=\left\{e_{1}, e_{2}, \ldots, e_{m}\right\}$.

Definition 1.1. A subset $C$ of $V$ is called a covering set of $G$ if every edge of $G$ is incident to at least one vertex of $C$. Any covering set with minimum cardinality is called a minimum covering set.

Definition 1.2. A subset of $E$ is called an edge covering set of $G$ if every vertex of $G$ is incident to at least one edge of it. Any edge covering set with minimum cardinality is called a minimum edge covering set. Let $C_{E}$ be a minimum edge covering set of a graph $G$. The minimum edge covering matrix of $G$ is a $m \times m$ matrix $A_{C_{E}}(G)=\left(e_{i j}\right)_{m \times m}$, where

$$
e_{i j}= \begin{cases}1, & \text { if } e_{i}, e_{j} \text { are adjacent } \\ 1, & \text { if } i=j \text { and } e_{i} \in C_{E} \\ 0, & \text { otherwise }\end{cases}
$$

The characteristic polynomial of $A_{C_{E}}(G)$ is denoted by $f_{m}(G, \lambda)=\operatorname{det}(\lambda I-$ $\left.A_{C_{E}}(G)\right)=c_{0} \lambda^{m}+c_{1} \lambda^{m-1}+c_{2} \lambda^{m-2}+\cdots+c_{m}$. The matrix $A_{C_{E}}(G)$ is real and symmetric. Then the eigenvalues of $A_{C_{E}}(G)$ are real numbers and are labeled in non-increasing order $\lambda_{1} \geq \lambda_{2} \geq \cdots \geq \lambda_{m}$. The minimum edge covering energy of $G$ is defined as

$$
E_{C_{E}}(G)=\sum_{i=1}^{m}\left|\lambda_{i}\right|
$$

\section{Problem Formulation and some Basic Properties of Minimum Edge COVERING ENERGY}

Following theorem obtains the coefficients of polynomial without applying determinant expansion. 
Theorem 2.1. Let $G$ be a graph with vertex set $V$, edge set $E$, and the minimum edge covering set $C_{E}$. Let $f_{m}(G, \lambda)=\operatorname{det}\left(\lambda I-A_{C_{E}}(G)\right)=c_{0} \lambda^{m}+c_{1} \lambda^{m-1}+c_{2} \lambda^{m-2}+\cdots+c_{m}$ be the characteristic polynomial of $G$. Then

(i) $c_{0}=1$;

(ii) $c_{1}=-\left|C_{E}\right|$;

(iii) $c_{2}=\left(\begin{array}{c}\left|C_{E}\right| \\ 2\end{array}\right)-\left(\frac{1}{2} \sum_{i=1}^{m} \operatorname{deg}\left(e_{i}\right)\right)$;

(iv) $c_{3}=-\left(\begin{array}{c}\left|C_{E}\right| \\ 3\end{array}\right)+\left|C_{E}\right|\left(\frac{1}{2} \sum_{i=1}^{m} \operatorname{deg}\left(e_{i}\right)\right)-\sum_{i \in C_{E}} \operatorname{deg}\left(e_{i}\right)-2\left(K_{1,3}+C_{3}\right)$, where $C_{3}$ is the number of triangles and $K_{1,3}$ is the number of star graphs with four vertices in $G$.

Proof. (i) Directly from the definition of $f_{m}(G, \lambda)$, it follows that $c_{0}=1$.

(ii) Since the sum of diagonal elements of $A_{C_{E}}(G)$ is equal to $\left|C_{E}\right|$, sum of determinants of all $1 \times 1$ principal submatrices of $A_{C_{E}}(G)$ is the trace of $A_{C_{E}}(G)$ which evidently equal to $\left|C_{E}\right|$. Thus, $(-1)^{1} c_{1}=\left|C_{E}\right|$.

(iii) $(-1)^{2} c_{2}$ is equal to sum of determinats of all the $2 \times 2$ principal submatrices of $A_{C_{E}}(G)$, that is

$$
\begin{aligned}
c_{2} & =\sum_{1 \leq i<j \leq m}\left|\begin{array}{cc}
e_{i i} & e_{i j} \\
e_{j i} & e_{j j}
\end{array}\right|=\sum_{1 \leq i<j \leq m}\left(e_{i i} e_{j j}-e_{i j} e_{j i}\right) \\
& =\sum_{1 \leq i<j \leq m} e_{i i} e_{j j}-\sum_{1 \leq i<j \leq m} e_{i j}^{2}=\left(\begin{array}{c}
\left|C_{E}\right| \\
2
\end{array}\right)-\frac{1}{2} \sum_{i=1}^{m} \operatorname{deg}\left(e_{i}\right) .
\end{aligned}
$$

(iv)

$$
\begin{aligned}
c_{3}= & (-1)^{3} \sum_{1 \leq i<j<k \leq m}\left|\begin{array}{ccc}
e_{i i} & e_{i j} & e_{i k} \\
e_{j i} & e_{j j} & e_{j k} \\
e_{k i} & e_{k j} & e_{k k}
\end{array}\right| \\
= & -\sum_{1 \leq i<j<k \leq m}\left[e_{i i}\left(e_{j j} e_{k k}-e_{k j} e_{j k}\right)-e_{i j}\left(e_{j i} e_{k k}-e_{k i} e_{j k}\right)\right. \\
& \left.+e_{i k}\left(e_{j i} e_{k j}-e_{k i} e_{j j}\right)\right] \\
= & -\sum_{1 \leq i<j<k \leq m} e_{i i} e_{j j} e_{k k}+\sum_{1 \leq i<j<k \leq m}\left[e_{i i} e_{j k}+e_{j j} e_{i k}+e_{k k} e_{i j}\right] \\
& -\sum_{1 \leq i<j<k \leq m} e_{i j} e_{j k} e_{k i}-\sum_{1 \leq i<j<k \leq m} e_{i k} e_{k j} e_{j i} \\
= & \left(\begin{array}{c}
\left|C_{E}\right| \\
3
\end{array}\right)+\left|C_{E}\right|\left(\frac{1}{2} \sum_{i=1}^{m} \operatorname{deg}\left(e_{i}\right)\right)-\sum_{i \in C_{E}} \operatorname{deg}\left(e_{i}\right)-2\left(k_{1,3}+C_{3}\right) .
\end{aligned}
$$

Thus,

$$
c_{3}=\left(\begin{array}{c}
\left|C_{E}\right| \\
3
\end{array}\right)+\left|C_{E}\right|\left(\frac{1}{2} \sum_{i=1}^{m} \operatorname{deg}\left(e_{i}\right)\right)-\sum_{i \in C_{E}} \operatorname{deg}\left(e_{i}\right)-2\left(k_{1,3}+C_{3}\right)
$$


Corollary 2.1. Let $G$ be a path $P_{n}$ with $n$ vertices and $m$ edges, then

(i) $c_{0}=1$;

(ii) $c_{1}=-\left\lceil\frac{m+1}{2}\right\rceil,\lceil x\rceil$ is the smallest integer number greater than or equals to $x$;

(iii) $c_{2}=\left(\begin{array}{c}\left\lceil\frac{m+1}{2}\right\rceil \\ 2\end{array}\right)-(m-1)$;

(iv) $c_{3}=\left(\begin{array}{c}\left\lceil\frac{m+1}{2}\right. \\ 3\end{array}\right)+\left\lceil\frac{m+1}{2}\right\rceil(m-1)-\sum_{i \in C_{E}} \operatorname{deg}\left(e_{i}\right)$.

Corollary 2.2. Let $G$ be a cycle $C_{n}$ with $n$ vertices and $m$ edges, then

(i) $c_{0}=1$;

(ii) $c_{1}=-\left\lceil\frac{m}{2}\right\rceil$;

(iii) $c_{2}=\left(\begin{array}{c}\left\lceil\frac{m}{2}\right. \\ 2\end{array}\right)-m$;

(iv) $c_{3}=-\left(\begin{array}{c}\left\lceil\frac{m}{2}\right. \\ 3\end{array}\right)+\left\lceil\frac{m}{2}\right\rceil(m-2), m \geq 4$.

Remark 2.1. Let $G$ be a path $P_{n}$ with $n$ vertices and $m$ edges, then

$$
\sum_{i=1}^{m} \operatorname{deg}\left(e_{i}\right)=2(m-1) \text {. }
$$

Theorem 2.2. If $\lambda_{1}, \lambda_{2}, \ldots, \lambda_{m}$ are eigenvalues of $A_{C_{E}}(G)$, then

$$
\sum_{i=1}^{m} \lambda_{i}^{2}=\left|C_{E}\right|+\sum_{i=1}^{m} \operatorname{deg}\left(e_{i}\right)
$$

Proof. The sum of squares of the eigenvalues of $A_{C_{E}}(G)$ is just the trace of $\left(A_{C_{E}}(G)\right)^{2}$ Therefore,

$$
\sum_{i=1}^{m} \lambda_{i}^{2}=\sum_{i=1}^{m} \sum_{j=1}^{m} e_{i j} e_{j i}=2 \sum_{i<j}\left(e_{i j}\right)^{2}+\sum_{i=1}^{m}\left(e_{i i}\right)^{2}=\left|C_{E}\right|+\sum_{i=1}^{m} \operatorname{deg}\left(e_{i}\right) .
$$

Theorem 2.3 (Parity theorem). Let $G$ be a graph with a minimum edge covering set $C_{E}$. If the minimum edge covering energy $E_{C_{E}}(G)$ of $G$ is a rational number, then

$$
E_{C_{E}} \equiv\left|C_{E}\right| \quad(\bmod 2) \text {. }
$$

Proof. Let $\lambda_{1}, \lambda_{2}, \ldots, \lambda_{r}$ be positive, and the rest of the minimum edge covering eigenvalues non-positive. Thus,

$$
E_{C_{E}}(G)=\sum_{i=1}^{m}\left|\lambda_{i}\right|=\left(\lambda_{1}+\lambda_{2}+\cdots+\lambda_{r}\right)-\left(\lambda_{r+1}+\lambda_{r+2}+\cdots+\lambda_{m}\right),
$$

implies $E_{C_{E}}(G)=2\left(\lambda_{1}+\lambda_{2}+\cdots+\lambda_{r}\right)-\left|C_{E}\right|$. Since $\lambda_{1}, \lambda_{2}, \ldots, \lambda_{r}$ are algebraic integers, so is their sum. Hence, $\lambda_{1}+\lambda_{2}+\cdots+\lambda_{r}$ must be an integer if $E_{C_{E}}(G)$ is rational. 


\section{The Minimum Edge Covering Energy of Some Graphs}

Theorem 3.1. The minimum edge covering energy of a star graph $K_{1, n-1}$ is $m$ for $n \geq 2$. The polynomial of it is $\lambda^{m}-m \lambda^{m-1}$.

Proof. Let $K_{1, n-1}$ be a star graph with vertex set $V=\left\{v_{0}, v_{1}, v_{2}, \ldots, v_{n-1}\right\}$ and center $v_{0}$, then

$$
A_{c_{E}}(G)=\left(\begin{array}{ccccc}
1 & 1 & 1 & \cdots & 1 \\
1 & 1 & 1 & \cdots & 1 \\
1 & 1 & 1 & \cdots & 1 \\
\vdots & \vdots & \vdots & \ddots & \vdots \\
1 & 1 & 1 & \cdots & 1
\end{array}\right)
$$

that $A_{c_{E}}(G)$ is $m \times m$. Then its characteristic polynomial is

$$
f_{m}\left(k_{1, n-1}, \lambda\right)=\operatorname{det}\left(\begin{array}{cccc}
\lambda-1 & -1 & \cdots & -1 \\
-1 & \lambda-1 & \cdots & -1 \\
\vdots & \vdots & \ddots & \vdots \\
-1 & -1 & \cdots & \lambda-1
\end{array}\right)
$$

By computing determinant of upper triangular of matrix (3.1) we will have

$$
f_{m}\left(k_{1, n-1}, \lambda\right)=\lambda^{m}-m \lambda^{m-1}
$$

Then

$$
\begin{aligned}
\operatorname{Spec}\left(k_{1, n-1}\right) & =\left(\begin{array}{cc}
m & 0 \\
1 & m-1
\end{array}\right), \\
E_{C_{E}}(G) & =\sum_{i=1}^{m}\left|\lambda_{i}\right|=m .
\end{aligned}
$$

Theorem 3.2. Let $G$ be a path or a cycle graph with a minimum edge covering set $C_{E}$. Then $E_{C_{E}}(G) \simeq 2 m-\left|C_{E}\right|$ or $\left|E_{C_{E}}(G)-\left(2 m-\left|C_{E}\right|\right)\right| \leq 1$.

Proof. Let $\lambda_{1}, \lambda_{2}, \ldots, \lambda_{r}$ be positive eigenvalues, and the rest of the minimum edge covering eigenvalues non-positive. Thus,

$$
E_{C_{E}}(G)=\sum_{i=1}^{m}\left|\lambda_{i}\right|=\left(\lambda_{1}+\lambda_{2}+\cdots+\lambda_{r}\right)-\left(\lambda_{r+1}+\lambda_{r+2}+\cdots+\lambda_{m}\right),
$$

implies $E_{C_{E}}(G)=2\left(\lambda_{1}+\lambda_{2}+\cdots+\lambda_{r}\right)-\left|C_{E}\right|$. Since $\lambda_{1}, \lambda_{2}, \ldots, \lambda_{r}$ are algebraic integers, so sum $\left(\lambda_{1}+\lambda_{2}+\cdots+\lambda_{r}\right) \simeq m$. Hence, $E_{C_{E}}(G) \simeq 2 m-\left|C_{E}\right|$.

Theorem 3.3 (Upper bound). Let $G$ be a graph with $n$ vertices, $m$ edges, and let $C_{E}$ be a minimum edge covering set of $G$. Then

$$
E_{C_{E}}(G) \leq \sqrt{m\left(\sum_{i=1}^{m} \operatorname{deg}\left(e_{i}\right)+\left|C_{E}\right|\right)} .
$$


Proof. Let $\lambda_{1} \geq \lambda_{2} \geq \lambda_{3} \cdots \geq \lambda_{m}$ be the eigenvalues of $A_{C_{E}}(G)$. Bearing in mind the Cauchy-Schwarz inequality

$$
\left(\sum_{i=1}^{m} a_{i} b_{j}\right)^{2} \leq\left(\sum_{i=1}^{m} a_{i}^{2}\right)\left(\sum_{i=1}^{m} b_{i}^{2}\right)
$$

we put $a_{i}=1$ and $b_{i}=\left|\lambda_{i}\right|$ and we have

$$
\left[E_{C_{E}}(G)\right]^{2}=\left(\sum_{i=1}^{m}\left|\lambda_{i}\right|\right)^{2} \leq m\left(\sum_{i=1}^{m}\left|\lambda_{i}\right|^{2}\right)=m \sum_{i=1}^{m} \lambda_{i}^{2}=m\left(\sum_{i=1}^{m} \operatorname{deg}\left(e_{i}\right)+\left|C_{E}\right|\right)
$$

Theorem 3.4 (Lower bound). Let $G$ be a graph with $n$ vertices and $m$ edges, and let $C_{E}$ be a minimum edge covering set of $G$. If $D=\left|\operatorname{det} A_{C_{E}}(G)\right|$, then

$$
\left[E_{C_{E}}(G)\right]^{2} \geq \sum_{i=1}^{m} \operatorname{deg}\left(e_{i}\right)+\left|C_{E}\right|+m(m-1) \sqrt[m]{\left(\prod_{i=1}^{m} \lambda_{i}\right)^{2}}
$$

with equality if $G$ is a star of order $n$.

Proof. We have

$$
\left[E_{C_{E}}(G)\right]^{2}=\left(\sum_{i=1}^{m}\left|\lambda_{i}\right|\right)^{2}=\left(\sum_{i=1}^{m}\left|\lambda_{i}\right|\right)\left(\sum_{i=1}^{m}\left|\lambda_{i}\right|\right)=\sum_{i=1}^{m}\left|\lambda_{i}\right|^{2}+\sum_{i \neq j}\left|\lambda_{i}\right|\left|\lambda_{j}\right| .
$$

Now, by inequality between the arithmetic mean and geometric mean, we have

$$
\frac{1}{m(m-1)} \sum_{i \neq j}\left|\lambda_{i}\right|\left|\lambda_{j}\right| \geq\left(\prod_{i \neq j}\left|\lambda_{i}\right|\left|\lambda_{j}\right|\right)^{\frac{1}{m(m-1)}} .
$$

Thus,

$$
\begin{aligned}
{\left[E_{C_{E}}(G)\right]^{2} } & \geq \sum_{i=1}^{m}\left|\lambda_{i}\right|^{2}+m(m-1)\left(\prod_{i \neq j}\left|\lambda_{i}\right|\left|\lambda_{j}\right|\right)^{\frac{1}{m(m-1)}} \\
& \geq \sum_{i=1}^{m}\left|\lambda_{i}\right|^{2}+m(m-1)\left(\prod_{i=1}^{m}\left|\lambda_{i}\right|^{2(m-1)}\right)^{\frac{1}{m(m-1)}} \\
& =\sum_{i=1}^{m}\left|\lambda_{i}\right|^{2}+m(m-1)\left|\prod_{i=1}^{m} \lambda_{i}\right|^{\frac{2}{m}} \\
& =\sum_{i=1}^{m} \operatorname{deg}\left(e_{i}\right)+\left|C_{E}\right|+m(m-1) D^{\frac{2}{m}}
\end{aligned}
$$

Since in the star graphs the multiplicity of $\lambda=0$ is $m-1$, so $\prod_{i=1}^{m} \lambda_{i}=\operatorname{det} A_{C_{E}}(G)=0$, $\left|C_{E}\right|=m$ and $\sum_{i=1}^{m} \operatorname{deg}\left(e_{i}\right)=m(m-1)$. Then, by placing the above values in (3.2), equality cases hold

$$
m^{2}=\left[E_{C_{E}}\left(K_{1, n-1}\right)\right]^{2} \geq m(m-1)+m+m(m-1) \sqrt[m]{\left(\prod_{i=1}^{m} \lambda_{i}\right)^{2}}=m^{2} .
$$




\section{ACKNOWLEDGEMENTS}

This research was supported by Research Council of Semnan University. The authors are grateful to Dr. Tatjana Aleksic Lampert and the anonymous reviewer for his/her valuable comments and suggestions helped to improve the quality of this work.

\section{REFERENCES}

[1] I. Gutman, The energy of a graph, Ber. Math-Statist. Sekt. Forschungsz. Graz 103 (1978), 1-22.

[2] I. Gutman, B. Furtula, E. Zogić and E. Glogić, Resolvent energy of graphs, MATCH Commun. Math. Comput. Chem. 75 (2016), 279-290.

[3] R. B. Bapat, Graphs and Matrices, Hindustan Book Agency, Springer, London, 2011.

[4] S. K. Vaidya and K. M. Popat, Some new results on energy of graphs, MATCH Commun. Math. Comput. Chem. 77 (2017), 589-594.

[5] V. Nikiforov, The energy of graphs and matrices, J. Math. Anal. Appl. 326 (2007), 1472-1475.

[6] A. Jahanbani, Lower bounds for the energy of graphs, AKCE Int. J. Graphs Comb. 5 (2018), 88-96.

[7] K. C. Das and I. Gutman, Bounds for the energy of graphs, Hacet. J. Math. Stat. 45(3) (2016), 695-703.

[8] C. Adiga, A. Bayad, I. Gutman and S. A. Srinivas, The minimum covering energy of graph, Kragujevac Journal of Science 34 (2012), 39-56.

[9] I. Gutman and S. Wagner, The matching energy of a graph, Applied Mathematics 160 (2012), $2177-2187$.

[10] J. Zhang, H. Kan and X. Liu, Graphs with extremal incident energy, Filomat 29(6) (2015), 1251-1258.

[11] M. Jooyandeh, D. Kiani and M. Mirzakhah, Incidence energy of a graph, MATCH Commun. Math. Comput. Chem. 62 (2009), 561-572.

[12] I. Gutman, D. Kiani and M. Mirzakhah, On incidence energy of a graph, MATCH Commun. Math. Comput. Chem. 62 (2009), 573-580.

[13] R. Kanna, B. N. Dharmendra and G. Sridhara, Laplacian minimum dominating energy of a graph, International Journal of Pure and Applied Mathematics 89(4) (2013), 565-581.

[14] R. Kanna, B. N. Dharmendra and G. Sridhara, Minimum dominating distance energy of a graph, J. Indian Math. Soc. (N.S.) 20 (2014), 19-29.

[15] R. Kanna, B. N. Dharmendra and G. Sridhara, Minimum dominating energy of a graph, International Journal of Pure and Applied Mathematics 85(4) (2013), 707-718.

[16] S. K. Vaidya and R. M. Pandit, Edge domination in some path and cycle related graphs, Hindawi Publishing Corporation, ISRN Discrete Mathematics (2014), Article ID 975812, 5 pages.

\footnotetext{
${ }^{1}$ Department of Mathematics, Statistics and Computer Science, Semnan University, AddressSemnan University, SEmnan, Iran

Email address: sabeti.samira@semnan.ac.ir

Email address: banihashemi.akram@semnan.ac.ir

Email address: s_mohammadian@semnan.ac.ir
} 